\title{
CONCENTRACIÓN DE CARBONO EN VÁSTAGOS Y RAÍCES DE DIFERENTES ESPECIES DE PASTOS EN NUEVO LEÓN, MÉXICO
}

\author{
CARBON CONCENTRATION IN SHOOTS AND ROOTS OF DIFFERENT \\ GRASS SPECIES IN NUEVO LEON, MEXICO
}

\author{
José I. Yerena-Yamallel*, Javier Jiménez-Pérez, \\ Eduardo Alanís-Rodríguez y Oscar A. Aguirre-Calderón
}

Universidad Autónoma de Nuevo León, Facultad de Ciencias Forestales, Linares, Nuevo León, México.

*Autor de correspondencia (israel.yerena@gmail.com)

\section{RESUMEN}

En México, los estudios sobre la evaluación del coeficiente de concentración de carbono en pastos son de gran importancia, pero no hay información al respecto. En el presente estudio, se determinó la concentración de $C$ en vástagos y raíces de 21 especies de pastos. El estudio se realizó durante los meses de abril y mayo de 2018 en los municipios de Linares y Montemorelos, Nuevo León, México. Se analizó un total de 378 muestras provenientes de 21 especies, tres sitios por especie, dos componentes por sitio y las mediciones se realizaron por triplicado. La concentración de $\mathrm{C}$ se obtuvo con el equipo Solids TOC Analyzer. La concentración promedio de $C$ en las especies varió de 32.5 \% en Paspalum notatum Flüggé a $39.9 \%$ en Setaria viridis (L.) P. Beauv. con media general de $36.4 \%$. La concentración de $C$ en el componente raíz de todas las especies fue $36.0 \%$ y en vástago $36.9 \%$. Pennisetum ciliare (L.) Link presentó la mayor concentración de $C$ en el vástago (41.9\%) y P. notatum mostró el valor menor (33.2\%). El contenido promedio de $\mathrm{C}$ en la raíz entre las especies varió de $31.8 \%$ en P. notatum a $39.8 \%$ en Cynodon plectostachyus (K. Schumann.) Pilg. Utilizar un valor generalizado para la concentración de carbono total da como resultado una sobrestimación del carbono capturado en los pastos.

Palabras clave: Paspalum notatum, Setaria viridis, matorral espinoso tamaulipeco, pastizal inducido

\section{SUMMARY}

In Mexico, studies on the evaluation of the carbon concentration coefficient in grasses are of great importance, but there is no information about it. In this study, the concentration of $\mathrm{C}$ in shoots and roots of 21 grass species was determined. The study was carried out during April and May 2018 in the municipalities of Linares and Montemorelos, Nuevo Leon, Mexico. A total of 378 samples was analyzed from 21 species, three sites per species, and two components per site; the measurements were made in triplicate. The concentration of $\mathrm{C}$ was obtained with the Solids TOC Analyzer. The average $C$ concentration in the species ranged from $32.5 \%$ in Paspalum notatum Flügge to 39.9 \% in Setaria viridis (L.) P. Beauv., with an overall average of $36.4 \%$. The concentration of $C$ in the root component of all the species was $36.0 \%$ and $36.9 \%$ in the shoot. Pennisetum ciliare (L.) Link showed the highest $C$ concentration in the shoot $(41.9 \%)$, and $P$. notatum displayed the lowest value (33.22\%). The average percentages of root $C$ content among the species ranged from $31.8 \%$ in P. notatum to $39.8 \%$ in Cynodon plectostachyus (K. Schumann.) Pilg. An overestimation of the carbon sequestered in grasses results from using a generalized value for total carbon concentration.

Index words: Paspalum notatum, Setaria viridis, induced grassland, Tamaulipan thorn scrub.

\section{INTRODUCCIÓN}

Las acciones preventivas que las políticas deben apoyar tienen que estar enfocadas en aumentar los beneficios de las medidas de adaptación y mitigación contra del cambio climático, mejorar los niveles de vida, los servicios de los ecosistemas y la seguridad alimentaria. Los pastizales están lejos de ser el eje de las negociaciones actuales del clima mundial, pero son importantes y merecen mayor atención. Los pastizales asumen potencial de mitigación (Dass et al., 2018) y son relevantes en la salud humana y ambiental, por lo que su manejo inadecuado tendría consecuencias negativas en la subsistencia (FAO, 2009).

Los pastizales constituyen los ecosistemas más extensos del mundo, cubren más del 40 \% de la superficie de la tierra (Hewins et al., 2018), representan a los biomas más productivos y diversos, se caracterizan por contener vegetación abierta dominada por especies herbáceas y su producción primaria es aprovechada directamente por los herbívoros (Deguignet et al., 2014; Newman, 2000; White et al., 2000).

Los ecosistemas forestales en general adquieren enorme potencial al funcionar como sumidero terrestre de carbono (Graciano-Ávila et al., 2019), y dentro de ellos, los pastizales son de especial importancia, pues se estima que secuestran de 0.1 a $0.6 \mathrm{Mg}$ por hectárea al año, lo que disminuye la acumulación del $\mathrm{CO}_{2}$ en la atmósfera e impacta en la temperatura del planeta (Jones y Donnelly, 2004). Según Costanza et al. (1997), si se otorgara un valor económico al servicio ambiental que generan los pastizales sobre el clima, éste sería de 1.2 US dólares por hectárea por año, y si se multiplica por el total de la superficie que ocupan en el mundo, se alcanzaría la cifra de 4677 millones de US dólares. Con un manejo adecuado, este tipo de ecosistema terrestre podría almacenar a nivel mundial entre 100 y $800 \mathrm{Mt}$ de $\mathrm{CO}_{2}$, y un total de 1752 a 
2385 Gt de C al año; de esta cantidad, 71 \% se encuentra debajo de la superficie del suelo (IPCC, 2007) y tiene el potencial biofísico para secuestrar de 1.3 a $2.0 \mathrm{Gt}$ de $\mathrm{CO}_{2}$ para el año 2030 (Smith et al., 2008), lo que proporcionaría importantes beneficios para la mitigación del cambio climático.

En México no hay estudios orientados a evaluar la concentración de carbono en especies de pastizales (Pompa-García y Sigala-Rodríguez, 2017). Si se tiene en cuenta que existen áreas extensas de pastizales en el noreste del país, es necesario realizar trabajos encaminados a la medición y dinámica del carbono en el ecosistema que exponga los beneficios al ambiente. En el presente estudio se planteó determinar la concentración de carbono en vástagos y raíces de 21 especies de pastos presentes en el matorral espinoso tamaulipeco, pastizal inducido, matorral submontano y bosque de encino. Las hipótesis planteadas fueron 1) que existen diferencias significativas en la concentración de carbono entre las especies y 2) entre los componentes.

\section{MATERIALES Y MÉTODOS}

\section{Área de estudio}

El estudio se realizó en cuatro comunidades vegetales (matorral espinoso tamaulipeco, pastizal inducido, matorral submontano y bosque de encino) de los municipios de Linares y Montemorelos, Nuevo León, México (coordenadas geográficas $24^{\circ} 47^{\prime} 23^{\prime \prime}$ a $25^{\circ} 02^{\prime}$ $08^{\prime \prime} \mathrm{N}$ y $99^{\circ} 31^{\prime} 37^{\prime \prime}$ a $99^{\circ} 54^{\prime} 38^{\prime \prime} \mathrm{O}$, con en un intervalo altitudinal de 350 a $700 \mathrm{~m}$ ).

En los sitios estudiados hay ausencia de ganado doméstico, pero se observaron indicios de fauna silvestre. El clima es $(A) C\left(x^{\prime}\right) w^{\prime \prime} a(e)$ correspondiente a un clima extremoso semi-cálido sub-húmedo, básicamente debido a la posición geográfica en la zona subtropical de altas presiones, con influencia de los vientos alisios húmedos y vientos fríos del norte durante el invierno. La precipitación pluvial anual oscila entre 400 y 1850 mm, con valor promedio de $805 \mathrm{~mm}$. La temperatura promedio anual es de $22.4^{\circ} \mathrm{C}$ con temperaturas mayores a los $40{ }^{\circ} \mathrm{C}$ en verano e inferiores a $0^{\circ} \mathrm{C}$ en invierno. Los suelos de la zona corresponden al típico vertisol de origen aluvio-coluvial.

\section{Selección de sitios de muestreo}

Mediante recorridos de campo se seleccionaron 21 especies de pastos (Cuadro 1) con base en su abundancia y con las características siguientes: 1) bien conservado, con poca o nula vegetación indicadora de disturbio, 2) con $100 \%$ de cobertura vegetal, 3) con presencia de especies características del tipo de vegetación, y 4) con condiciones que permitan efectuar las mediciones correspondientes. El tipo de muestreo en campo fue dirigido; es decir, solamente se recolectó la especie de interés, se seleccionaron tres sitios de muestreo por especie a una distancia mínima de separación de 100 m, lo que sumó 63 sitios en todo el experimento. Los sitios se establecieron en un espacio de forma cuadrangular de $30 \times 30 \mathrm{~cm}$.

\section{Preparación de las muestras}

Para la colecta de cada muestra se utilizó el método destructivo aplicado por Castro et al. (2011), el cual estima con precisión el rendimiento de forrajes. El muestreo en campo se realizó en los meses de abril y mayo de 2018 , momento en que las plantas alcanzan el desarrollo y crecimiento completo de la parte aérea y radical; para extraer esta última del suelo se utilizó una pala recta, cuidando de no cortar las raíces; se retiró el resto de tierra y se lavaron las raíces; la plantas completas se introdujeron en bolsas de papel con etiquetas de identificación y fueron trasladadas al laboratorio de carbono de la Facultad de Ciencias Forestales de la UANL, se colocaron a la sombra por $24 \mathrm{~h}$ para un pre-secado de la muestra (Quispe et al., 2015); el vástago se separó de la raíz haciendo un corte a nivel del cuello de la planta con tijeras de podar de mano.

Cada componente de la planta se pesó por separado y colocó en una estufa de secado marca VWR ${ }^{\circledR}$ modelo 1305U (WR International, Leuven, Bélgica) a $76{ }^{\circ} \mathrm{C}$ hasta alcanzar peso constante; a continuación, cada componente por planta se trituró en un molino pulverizador marca Fritsch ${ }^{\circledR}$ modelo Pulverisette 2 (Fritsch Inc, Oberstein, Alemania) hasta quedar en fracciones menores de $10 \mu \mathrm{m}$, las cuales fueron depositadas en bolsas de plástico, previa identificación; para evitar contaminación entre muestras se limpió el tazón al moler cada componente (Jiménez et al., 2013).

\section{Determinación de carbono}

La concentración de carbono se determinó con el equipo analítico Solids TOC Analyzer (OI AnalyticalSRA Instruments, Cernusco sul Naviglio, Italia), que actúa mediante la combustión completa de la muestra sólida a temperaturas de $900{ }^{\circ} \mathrm{C}$; los gases producto de la combustión pasan por una cámara con detector infrarrojo no dispersivo que contabiliza las moléculas de carbono contenidas en estos gases. Esta metodología fue utilizada por Rodríguez-Laguna et al. (2009) para especies maderables en un estudio de estimación de carbono almacenado en el bosque de pino-encino en la reserva de la biosfera El Cielo, Tamaulipas, México. El intervalo de detección de concentración de carbono en las muestras 
Cuadro 1. Especies de pasto presentes en el matorral espinoso tamaulipeco, pastizal inducido, matorral submontano y bosque de encino en Linares y Montemorelos, Nuevo León, México.

\begin{tabular}{ll}
\hline Nombre científico & Nombre común \\
\hline Dichanthium annulatum (Forssk.) Stapf & Zacate angleton pretoria \\
Panicum maximum Jacq. & Zacate guinea \\
Bothriochloa wrightii (Hackel) Henrard. & Popotillo cola de coyote \\
Cenchrus echinatus L. & Zacate cadillo \\
Panicum hallii Vasey & Panizo rizado \\
Sorghum halepense (L.) Pers. & Zacate johnson \\
Eustachys petraea (Sw.) Desv. & Pasto del dedo \\
Pennisetum ciliare (L.) Link & Zacate buffel \\
Bouteloua curtipendula (Michx.) Torr. & Banderilla \\
Bothriochloa ischaemum (L.) Keng & Tallo azul de kingranch \\
Brachiaria brizantha (Hochst. ex A. Rich.) Stapf & Insurgente \\
Setaria viridis (L.) P. Beauv. & Almorejo \\
Cynodon dactylon (L.) Pers. & Zacate bermuda \\
Eleusine indica (L.) Gaertn. & Pata de gallo \\
Paspalum notatum Flüggé & Pasto bahía \\
Aristida adscensionis L. & Tres barbas \\
Andropogon gerardii Vitman. & Tallo azul \\
Cynodon plectostachyus (K. Schumann.) Pilg. & Pasto estrella \\
Cyperus esculentus L. & Coquillo amarillo \\
Melinis repens (Willd.) Zizka & Pasto rosado \\
Cyperus rotundus L. & Coquillo rojo \\
\hline
\end{tabular}

oscila de $50 \mu \mathrm{g}$ a $30 \mathrm{mg}$ de carbono, con una precisión de $\pm 15 \mu \mathrm{g}$. Se realizaron tres análisis por especie para cada componente de vástago y raíz, con la finalidad de obtener el valor promedio (Yerena et al., 2012). En total se analizaron 378 muestras, las cuales se obtuvieron de muestreos de tres sitios por cada una de las 21 especies y de los dos componentes morfológicos mencionados, además de que las determinaciones se realizaron por triplicado.

\section{Análisis estadístico}

El análisis estadístico de los datos se efectuó mediante el programa Statistica Advanced. Dos factores se consideraron: especie y componente; dentro del factor especie hubo 21 niveles, y en el factor componente dos niveles (vástago y raíz). Cuando los datos cumplieron con los criterios de normalidad y homocedasticidad se realizó análisis de varianza $(P \leq 0.05)$ bajo un modelo completamente aleatorio. A las variables que presentaron diferencias significativas se les aplicó la prueba de comparación de medias de Tukey $(P \leq 0.05)$.

\section{RESULTADOS Y DISCUSIÓN}

\section{Concentración de carbono por especie}

La concentración de C presente en la planta completa mostró diferencias significativas $(P \leq 0.0001)$ entre especies, por lo cual la primera hipótesis se acepta. Los pastos conocidos como almorejo y zacate johnson (Setaria viridis y Sorghum halepense) presentaron los mayores valores, con 39.9 y $39.5 \%$, respectivamente; en cambio, el pasto bahía (Paspalum notatum) registró la menor concentración de carbono con 32.5 \%; la media general fue de $36.4 \%$ (Cuadro 2); dicha diferencia se atribuye a que las especies tienen variación en sus contenidos de proteína, celulosa, hemicelulosa y lignina (Del Pozo, 2002). El intervalo de variación en la concentración de carbono entre las especies fue de $7.4 \%$, mientras que para hierbas de pastizales en otras regiones del país, como la Sierra Norte de Oaxaca, se reportó una diferenciación superior de 17.3 puntos porcentuales, de 25.1 a 42.4 \% (FigueroaNavarro et al., 2005), al igual que para herbáceas del 
bosque de oyamel del Parque Nacional el Chico en el estado de Hidalgo, el intervalo fue de 38 a 50 \% (Razo et al., 2015), debido a las condiciones climáticas, latitud, edad de la planta, capacidad fotosintética, composición química y tipo de suelo (Irving, 2015; Ma et al., 2018).

La concentración de carbono por especie resultó en todos los casos inferior al valor de 50 \% sugerido por el IPCC (2006) para los tejidos vegetales. Gasca et al. (2014) reportaron $37 \%$ para el pasto Brachiaria brizantha en Colombia, porcentaje similar al del presente estudio. Andrade et al. (2008) reportaron $43.7 \%$ para la misma especie en Costa Rica. Es probable que las causas de esta variación entre las regiones geográficas sean que la especie de este estudio se desarrolló en una zona semiárida, mientras que la de Costa Rica se estableció en zona tropical, ambientes discrepantes en temperatura, iluminación y disponibilidad de agua, lo que causó diferencias en sus características anatómicas, en procesos fisiológicos como la fotorrespiración, carboxilación y oxigenación, así como en las actividades de las enzimas (Gliessman,
2002); sin embargo, fue inconsistente con los resultados de un estudio para diferentes regímenes climáticos donde la concentración de carbono en hierbas y pastos presentó una tendencia similar, seco $42.1 \%$, frío 42.0 \% y húmedo 41.6 \% (Jain et al., 2010). Los datos de especies en particular contribuyeron a mejorar la precisión en las estimaciones del balance de carbono en los ecosistemas terrestres, lo que redujo la incertidumbre en los inventarios de carbono (Pompa-García et al., 2017)

\section{Concentración de carbono por componente}

El análisis de varianza presentó diferencias no significativas $(P \leq 0.080352)$ en la concentración de carbono entre los componentes de la planta, por lo que la segunda hipótesis se rechaza. El componente radical presentó en promedio $36.0 \pm 2.8 \%$ y el vástago $36.9 \pm 2.7 \%$. La manera en que las plantas distribuyen los compuestos de carbono derivados de la fotosíntesis y los destinan para los diferentes procesos fisiológicos y componentes vegetales es descrita mediante el término repartición de

Cuadro 2. Concentración de carbono en especies de pasto presentes en el matorral espinoso tamaulipeco, pastizal inducido, matorral submontano y bosque de encino, en Linares y Montemorelos, Nuevo León, México.

\begin{tabular}{lcc}
\hline Especie & Media $\pm \mathrm{DE}$ & Agrupación Tukey \\
\hline Paspalum notatum & $32.5 \pm 0.6$ & $\mathrm{a}$ \\
Panicum maximum & $33.4 \pm 1.3$ & $\mathrm{ab}$ \\
Eleusine indica & $34.0 \pm 0.5$ & $\mathrm{abc}$ \\
Cyperus rotundus & $34.6 \pm 1.0$ & $\mathrm{abcd}$ \\
Dichanthium annulatum & $34.9 \pm 1.0$ & abcdf \\
Cenchrus echinatus & $34.9 \pm 0.8$ & abcdf \\
Bothriochloa wrightii & $35.1 \pm 1.1$ & abcdfg \\
Melinis repens & $35.3 \pm 1.1$ & abcdfgh \\
Bothriochloa ischaemum & $35.8 \pm 0.6$ & bcdfghi \\
Panicum hallii & $36.8 \pm 1.1$ & cdfghij \\
Aristida adscensionis & $36.9 \pm 0.9$ & $\mathrm{dfghij}$ \\
Andropogon gerardii & $36.9 \pm 0.3$ & $\mathrm{dfghij}$ \\
Cyperus esculentus & $37.1 \pm 1.1$ & $\mathrm{dfghijk}$ \\
Brachiaria brizantha & $37.3 \pm 1.6$ & $\mathrm{dfghijk}$ \\
Cynodon dactylon & $37.5 \pm 0.6$ & fghijk \\
Eustachys petraea & $37.9 \pm 1.2$ & ghijk \\
Cynodon plectostachyus & $38.0 \pm 0.4$ & hijk \\
Bouteloua curtipendula & $38.5 \pm 0.6$ & ijk \\
Pennisetum ciliare & $38.6 \pm 0.01$ & ijk \\
Sorghum halepense & $39.5 \pm 0.0$ & jk \\
Setaria viridis & $39.9 \pm 0.7$ & $\mathrm{k}$ \\
\hline DE & & \\
\hline
\end{tabular}

DE: desviación estándar. Medias con letras distintas son estadísticamente diferentes (Tukey, P $\leq 0.05$ ). 
carbono, que involucra la fuente, la vía y un destino (Del Pozo, 2002; Irving, 2015). Durante el crecimiento, varias estructuras de la planta compiten entre sí para obtener parte del carbono fijado por las hojas, y como resultado, algunas partes acumulan más biomasa que otras (Gliessman, 2002).

\section{Concentración de carbono en el vástago}

Para la concentración de carbono en el vástago de las plantas la diferencia fue significativa $(P \leq 0.0001)$ entre especies. Las especies de pasto Setaria viridis, Sorghum halepense, Cyperus esculentus, Cynodon dactylon y Pennisetum ciliare tuvieron mayor concentración de carbono en el vástago, con valores desde 40.0 hasta 41.9 $\%$; en contraste, las especies Paspalum notatum, Panicum maximum, Cyperus rotundus, Eleusine indica, Melinis repens, Brachiaria brizantha, Bothriochloa ischaemum, Dichanthium annulatum, Cynodon plectostachyus, Bothriochloa wrightii, Eustachys petraea y Panicum hallii tuvieron los valores menores, desde 33.2 hasta $36.7 \%$ (Cuadro 3).
Existen diferencias entre especies que poseen vías bioquímicas similares para la fotosíntesis en cuanto a la actividad fotosintética, crecimiento y desarrollo, atribuyéndose ésto a las diferentes características anatómicas, metabólicas y asimilación del carbono que presentan los follajes en las plantas de pastos (Del Pozo, 2002).

La concentración de carbono en el vástago de las 21 especies fue mayor a lo reportado por Yaranga y Custodio (2013) para cuatro especies de pastos naturales altoandinos de la provincia de Huancayo, Junín, Perú, con valores entre 29.1 y $32.1 \%$.

\section{Concentración de carbono en la raíz}

La concentración promedio de carbono en la raíz de los pastos presentó diferencias significativas $(P \leq 0.0001)$ entre especies. La concentración de carbono en la raíz de Paspalum notatum, Cenchrus echinatus, Panicum maximum, Dichanthium annulatum, Cyperus esculentus,

Cuadro 3. Concentración de carbono en el vástago de especies de pasto presentes en el matorral espinoso tamaulipeco, pastizal inducido, matorral submontano y bosque de encino, en Linares y Montemorelos, Nuevo León, México.

\begin{tabular}{lcc}
\hline Especie & Media \pm DE & Agrupación Tukey \\
\hline Paspalum notatum & $33.2 \pm 0.1$ & a \\
Panicum maximum & $33.5 \pm 0.3$ & ab \\
Cyperus rotundus & $33.7 \pm 0.6$ & abc \\
Eleusine indica & $33.8 \pm 0.5$ & abcd \\
Melinis repens & $34.2 \pm 1.1$ & abcde \\
Brachiaria brizantha & $35.1 \pm 0.7$ & abcde \\
Bothriochloa ischaemum & $36.0 \pm 1.4$ & abcde \\
Dichanthium annulatum & $36.2 \pm 1.9$ & abcde \\
Cynodon plectostachyus & $36.3 \pm 1.6$ & abcde \\
Bothriochloa wrightii & $36.3 \pm 1.8$ & abcde \\
Eustachys petraea & $36.5 \pm 0.8$ & abcdef \\
Panicum hallii & $36.7 \pm 1.0$ & abcdefg \\
Aristida adscensionis & $37.1 \pm 1.9$ & bcdefgh \\
Andropogon gerardii & $37.4 \pm 0.4$ & cdefgh \\
Cenchrus echinatus & $37.4 \pm 0.2$ & defgh \\
Bouteloua curtipendula & $37.6 \pm 0.3$ & efgh \\
Setaria viridis & $40.0 \pm 1.7$ & fghi \\
Sorghum halepense & $40.3 \pm 1.7$ & ghi \\
Cyperus esculentus & $40.6 \pm 2.0$ & hi \\
Cynodon dactylon & $40.8 \pm 1.2$ & hi \\
Pennisetum ciliare & $41.9 \pm 0.2$ & i \\
\hline EE: & &
\end{tabular}

DE: desviación estándar. Medias con letras distintas son estadísticamente diferentes (Tukey, P $\leq 0.05$ ). 
Bothriochloa wrightii, Cynodon dactylon, Eleusine indica, Pennisetum ciliare, Cyperus rotundus, Bothriochloa ischaemum, Andropogon gerardii, Melinis repens y Aristida adscensionis varió desde 31.8 hasta 36.6 \% (Cuadro 4). La concentración de carbono promedio en la raíz de la especie Paspalum notatum fue menor que la reportada por Yaranga y Custodio (2013) para Stipa ichu en Perú, de $32.2 \%$. La variación de la concentración de carbono entre los órganos se asocia con diferencias en sus composiciones químicas, los componentes de las plantas están desarrollados de varios compuestos orgánicos con diferentes concentraciones de C, como lignina con $63 \%$, celulosa con $44 \%$ y carbohidratos no estructurales con 44 $\%$ (Ma et al., 2018).

\section{CONCLUSIONES}

La concentración de carbono en plantas de 21 especies de pastos presentes en el matorral espinoso tamaulipeco, pastizal inducido, matorral submontano y bosque de encino, en Linares y Montemorelos, Nuevo León, México fueron significativamente diferentes entre ellas; la media general de concentración de carbono fue de $36.4 \%$ y el intervalo en plantas completas fue desde 32.5 a 39.9, en pasto bahía (Paspalum notatum) y pasto almorejo (Setaria viridis), respectivamente. Al comparar la concentración de carbono del vástago y la raíz los resultados fueron similares, pero al analizar la concentración de carbono entre las especies, el vástago y la raíz mostraron valores significativamente diferentes. Generalizar la concentración de carbono total proporciona sobrestimación del carbono capturado en los pastos, por lo cual se deben utilizar valores para cada especie.

\section{AGRADECIMIENTOS}

Al Programa de Apoyo a la Investigación Científica y Tecnológica de la Universidad Autónoma de Nuevo León, por el apoyo financiero otorgado al proyecto "Concentración de carbono en pastos de diferentes sistemas de uso de suelo".

Cuadro 4. Concentración de carbono en la raíz de especies de pasto presentes en el matorral espinoso tamaulipeco, pastizal inducido, matorral submontano y bosque de encino, en Linares y Montemorelos, Nuevo León, México.

\begin{tabular}{|c|c|c|}
\hline Especie & Media $\pm \mathrm{DE}$ & Agrupación Tukey \\
\hline Paspalum notatum & $31.8 \pm 1.2$ & $a$ \\
\hline Cenchrus echinatus & $32.3 \pm 1.5$ & $a b$ \\
\hline Panicum maximum & $33.4 \pm 2.4$ & $a b$ \\
\hline Dichanthium annulatum & $33.6 \pm 0.6$ & $a b$ \\
\hline Cyperus esculentus & $33.7 \pm 0.6$ & $a b$ \\
\hline Bothriochloa wrightii & $33.8 \pm 0.7$ & $a b c$ \\
\hline Cynodon dactylon & $34.2 \pm 1.7$ & $a b c$ \\
\hline Eleusine indica & $34.2 \pm 0.6$ & $a b c$ \\
\hline Pennisetum ciliare & $35.3 \pm 0.2$ & abcd \\
\hline Cyperus rotundus & $35.5 \pm 2.1$ & abcd \\
\hline Bothriochloa ischaemum & $35.6 \pm 0.2$ & abcd \\
\hline Andropogon gerardii & $36.4 \pm 0.4$ & abcd \\
\hline Melinis repens & $36.5 \pm 1.2$ & abcd \\
\hline Aristida adscensionis & $36.6 \pm 0.3$ & abcd \\
\hline Panicum hallii & $36.9 \pm 2.0$ & bcd \\
\hline Sorghum halepense & $38.7 \pm 0.4$ & $c d$ \\
\hline Eustachys petraea & $39.2 \pm 3.0$ & $d$ \\
\hline Bouteloua curtipendula & $39.7 \pm 1.3$ & $d$ \\
\hline Brachiaria brizantha & $39.39 \pm 3.8$ & $d$ \\
\hline Setaria viridis & $39.7 \pm 1.7$ & $d$ \\
\hline Cynodon plectostachyus & $39.8 \pm 0.9$ & $d$ \\
\hline
\end{tabular}

DE: desviación estándar. Medias con letras distintas son estadísticamente diferentes (Tukey, $\mathrm{P} \leq 0.05$ ). 


\section{BIBLIOGRAFÍA}

Andrade H. J., R. Brook and M. Ibrahim (2008) Growth, production and carbon sequestration of silvopastoral systems with native timber species in the dry lowland of Costa Rica. Plant and Soil 308:11-22, https://doi.org/10.1007/s11104-008-9600-x

Castro R. R., A. Hernández G., G. Aguilar B. y 0. Ramírez R. (2011) Comparación de métodos para estimar rendimiento de forraje en praderas asociadas. Naturaleza y Desarrollo 9:38-46.

Costanza R., R. d'Arge, R. de Groot, S. Farber, M. Grasso, B. Hannon, ... and M. van den Belt (1997) The value of the world's ecosystems services and natural capital. Nature 387:253-260, https://doi org/ 10.1038/387253a0

Dass P., B. Z. Houlton, Y. Wang and D. Warlind (2018) Grasslands may be more reliable carbon sinks than forests in California Environmental Research Letters 13:074027, https://doi. org/10.1088/1748-9326/aacb39

Deguignet M., D. Juffe-Bignoli, J. Harrison, B. MacSharry, N. Burgess and N. Kingston (2014) 2014 United Nations List of Protected Areas. United Nations Environment Programme-World Conservation Monitoring Centre. Cambridge, UK. $30 \mathrm{p}$.

Del Pozo R. P. P. (2002) Bases ecofisiológicas para el manejo de los pastos tropicales. Pastos 32:109-137.

FAO, Food and Agriculture Organization (2009) Grasslands: enabling their potential to contribute to greenhouse gas mitigation. Food and Agriculture Organization of the United Nations. Rome, Italy. http://www.fao.org/fileadmin/templates/agphome/ documents/climate/FinalUNFCCCgrassland.pdf (February 2018)

Figueroa-Navarro C., J. D. Etchevers-Barra, A. Velázquez-Martínez y M. Acosta-Mireles (2005) Concentración de carbono en diferentes tipos de vegetación de la Sierra Norte de Oaxaca. Terra Latinoamericana 23:57-64.

Gasca S. C., E. H. Pérez y A. Figueroa C. (2014) Evaluación del potencial de captura de carbono en un sistema pastoril de Brachiaria brizantha, en la subcuenca alta del río Las Piedras y el municipio de Popayán. Suelos Ecuatoriales 44:42-50.

Gliessman S. R. (2002) Agroecología: Procesos Ecológicos en Agricultura Sostenible. LITOCAT. Turrialba, Costa Rica. 359 p.

Graciano-Ávila G., E. Alanís-Rodríguez, 0. A. Aguirre-Calderón, M. A. González-Tagle, E. J. Treviño-Garza, A. Mora-Olivo y E. BuendíaRodríguez (2019) Estimación de volumen, biomasa y contenido de carbono en un bosque de clima templado-frío de Durango, México. Revista Fitotecnia Mexicana 42:119-127, https://doi org/10.35196/rfm.2019.2.119-127

Hewins D. B., M. P. Lyseng, D. F. Schoderbek, M. Alexander, W. D. Willms, C. N. Carlyle, ... and E. W. Bork (2018) Grazing and climate effects on soil organic carbon concentration and particle-size association in northern grasslands. Scientific Reports 8:1336, https://doi org/10.1038/s41598-018-19785-1

IPCC, Intergovernmental Panel on Climate Change (2006) 2006 IPPC Guidelines for National Greenhouse Gas Inventories. Intergovernmental Panel on Climate Change Cambridge UK and New York, USA. https://www.ipcc-nggip.iges.or.jp/ public/2006gl/ (January 2018)

IPCC, Intergovernmental Panel on Climate Change (2007) Climate Change 2007: The Physical Science Basis. Contribution of the Working Group I to the Fourth Assessment Report of the Intergovernmental Panel on Climate Change. S. Solomon, D. Qin, M. Manning, Z. Chen, M. Marquis, K.B. Averyt, M. Tignor and H. L. Miller (eds.). Cambridge University Press. New York USA. $996 \mathrm{p}$

Irving L. J. (2015) Carbon assimilation, biomass partitioning and productivity in grasses. Agriculture 5:1116-1134, https:// doi:10.3390/agriculture5041116

Jain T. B., R. T. Graham and D. Adams (2010) Carbon concentrations and carbon pool distributions in dry, moist, and cold mid-aged forests of the Rocky Mountains. In: Integrated Management of Carbon Sequestration and Biomass Utilization Opportunities in a Changing Climate. T. B. Jain, R. T. Graham and J. Sandquist (eds.). Proceedings of the 2009 National Silviculture Workshop 15-18 June 2009. Department of Agriculture, Forest Service. Rocky Mountain Research Station. Fort Collins, Colorado, USA pp:39-59.

Jiménez P. J., E. J. Treviño G. y J. I. Yerena Y. (2013) Concentración de carbono en especies del bosque de pino-encino en la Sierra Madre Oriental. Revista Mexicana de Ciencias Forestales 4:50 61, https://doi.org/10.29298/rmcf.v4i17.420

Jones M. B. and A. Donnelly (2004) Carbon sequestration in temperate grassland ecosystems and the influence of management, climate and elevated $\mathrm{CO}_{2}$. New Phytologist 164:423-439, https://doi.org/10.1111/j.1469-8137.2004.01201.x

Ma S., F. He, D. Tian, D. Zou, Z. Yan, Y. Yang, ... and J. Fang (2018) Variations and determinants of carbon content in plants: a global synthesis Biogeosciences 15:693-702, https://doi.org/10.5194/bg-15693-2018

Newman E. I. (2000) Applied Ecology and Environmental Management. Second edition. Wiley-Blackwell. London, UK. 408 p, https://doi. org/10.1002/9780470698723

Pompa-García M. and J. A. Sigala -Rodríguez (2017) Variation of carbon uptake from forest species in Mexico: a review. Madera y Bosques 23:225-235, https://doi.org/10.21829/ myb.2017.2321512

Pompa-García M., J. A. Sigala-Rodríguez, E. Jurado and J. Flores (2017) Tissue carbon concentration of 175 Mexican forest species. iForest - Biogeosciences and Forestry 10:754-758, https://doi org/10.3832/ifor2421-010

Quispe N. C. M., F. Quispe C. y R. M. Yaranga C. (2015) Almacenamiento de carbono en pastos naturales de la subcuenca del Canipaco Huancayo. Apuntes de Ciencia y Sociedad 5:218-227, https:// doi.org/10.18259/acs.2015032

Razo Z. R., A. J. Gordillo M., R. Rodríguez L., C. C. Maycotte M. y 0. A Acevedo S. (2015) Coeficientes de carbono para arbustos y herbáceas del bosque de oyamel del Parque Nacional El Chico. Revista Mexicana de Ciencias Forestales 6:58-67, https://doi. org/10.29298/rmcf.v6i31.195

Rodríguez-Laguna R., J. Jiménez-Pérez, O. A. Aguirre-Calderón, E. J. TreviñoGarza y R. Razo-Zárate (2009) Estimación de carbono almacenado en el bosque de pino-encino en la Reserva de la Biosfera el Cielo, Tamaulipas, México. Ra Ximhai 5:317-327, https://doi org/10.35197/rx.05.03.2009.06.rr

Smith P., D. Martino, Z. Cai, D. Gwary, H. Janzen, P. Kumar, ... and J. Smith (2008) Greenhouse gas mitigation in agriculture. Philosophical Transactions of the Royal Society B 363:789-813, https://doi. org/10.1098/rstb.2007.2184

White R. P., S. Murray and M. Rohweder (2000) Pilot Analysis of Global Ecosystems: Grassland Ecosystems. World Resources Institute. Washington, DC, USA. $70 \mathrm{p}$.

Yaranga C. R. M. y M. Custodio V. (2013) Almacenamiento de carbono en pastos naturales altoandinos. Scientia Agropecuaria 4:313-319, https://doi.org/10.17268/sci.agropecu.2013.04.05

Yerena Y. J. I., J. Jiménez P., O. A. Aguirre C. y E. J. Treviño G. (2012) Contenido de carbono total en los componentes de especies arbóreas y arbustivas en áreas con diferente uso, en el matorral espinoso tamaulipeco, en México. Bosque 33:145-152, https:// doi.org/10.4067/S0717-92002012000200004 
